\section{Spender haben kein erhöhtes Gesundheitsrisiko}

Quelle: Ibrahim HN, Foley R, Tan L et al. Long-term consequences of kidney donation. N Engl J Med 2009; 360: 459-469

Thema: Lebendspenden machen etwa ein Viertel aller Nierenspenden aus. Problematisch könnte es für den Spender dann werden, wenn er einmal selbst nierenkrank wird. Die allgemeine Evidenzlage zeigt zwar, dass Lebendnierenspender eine ähnliche Überlebensrate wie Nichtspender und ein ähnliches Risiko für die Entwicklung einer „endstage renal disease“ (ESRD) haben, konkrete Zahlen dazu, wie groß das Gesundheitsrisiko ist, gab es bislang jedoch nicht.

Projekt: Diese Lücke haben Ibrahim et al. mit ihrer retrospektiven Studie nun geschlossen. Sie werteten die Daten von 3698 Spendern aus, die zwischen 1963 und 2007 eine Niere gespendet hatten. Die Wissenschaftler gingen der Frage nach, wie viele der Spender eine terminale Niereninsuffizienz entwickelten. Zusätzlich überprüften sie die glomeruläre Filtrationsrate (GFR) sowie den Albumingehalt im Urin und bestimmten die Prävalenz von Bluthochdruck, den Gesundheitszustand und die Lebensqualität von 255 Spendern.

Ergebnis: Die Überlebensrate der Nierenspender war ähnlich der nach Alter, Geschlecht und Rasse bzw. ethnischer Gruppe adjustierten Kontrollgruppe. Bei 11 Spendern manifestierte sich eine ESRD. Mit einer Häufigkeit von 180 Fällen in 1 Million Personen pro Jahr lag das Risiko sogar unter dem der Normalbevölkerung (268 Fälle pro Million Personenjahre).
Nach durchschnittlich 12 Jahren hatten 85,5\% der Spender aus der Subgruppe eine GFR von mehr als $60 \mathrm{ml} / \mathrm{min}$ pro $1,73 \mathrm{~m}^{2}$ Körperfläche, 32,1 \% litten an Bluthochdruck und $12,7 \%$ hatten eine Albuminurie - die Spender hatten also im Vergleich zur Normalbevölkerung kein erhöhtes Gesundheitsrisiko. Die meisten Nierenspender hatten sogar eine höhere Lebensqualität als die Normalbevölkerung.

Fazit: Die Überlebensrate und das Risiko für eine dialysepflichtige Niereninsuffizienz bei Lebendnierenspendern ähneln denen der Allgemeinbevölkerung. Die meisten untersuchten Spender hatten eine normale GFR bzw. Albuminexkretion und eine exzellente Lebensqualität. Dies liegt möglicherweise daran, dass die ausgewählten Spender nicht den Durchschnitt der Bevölkerung repräsentieren (relativ junge, weiße Amerikaner, kein Übergewicht, kein Hypertonus). Auf die immer älter werdenden Nierenspender kann man das Ergebnis daher nur bedingt übertragen.

Key words: Transplantation - Lebendnierenspender - ESRD - GFR - Albuminexkretion - Lebensqualität 